\title{
Clinical Analysis of Fractional Exhaled and Nasal Nitric Oxide in Allergic Rhinitis Children
}

\author{
Dabo Liu ${ }^{1^{*}}$, Zhenyun Huang ${ }^{1}$ and Yaping Huang \\ Department of ENT, Guangzhou women and children's medical centre, China \\ ${ }^{1}$ Equal contributors
}

*Corresponding author: Dabo Liu, Department of ENT, Guangzhou women and children's medical centre, China, Tel: 020-81330660; E-mail: daboliu@126.com

Received Date: January 27, 2015; Accepted Date: February 26, 2015; Published Date: March 06, 2015

Copyright: $\odot 2015$ Liu D et al. This is an open-access article distributed under the terms of the Creative Commons Attribution License, which permits unrestricted use, distribution, and reproduction in any medium, provided the original author and source are credited.

\begin{abstract}
The primary aim of this study was to assess whether Fractional Exhaled Nitric Oxide (FeNO) and Nasal Nitric Oxide ( $\mathrm{nNO}$ ) can be used as a mean of diagnosis in Allergic Rhinitis (AR) without asthma or not, and discuss their correlation. Fifteen healthy children and thirty children with AR, but without asthma, were enrolled. A questionnaire about clinical features of AR children had been completed by parents. The levels of FeNO and nNO were measured by NIOX MINO (Aerocrine AB, Solna, Sweden).IBM SPSS statistics 20.0 software was used to analyse the data.
\end{abstract}

Keywords: Fractional exhaled Nitric Oxide; Nasal Nitric Oxide; Atopy; Inflammation

\section{Introduction}

Airway inflammation resulting from the activation of mast cells and antigen-specific type $2 \mathrm{~T}$-helper cells (Th2), results in the production of cytokines, including interleukin (IL)-4, IL-5, and IL-13. In turn, IL-4 and IL-13 cause epithelial Inducible NO Synthase (iNOS) expression, which generates NO [1].Measurement of FeNO is a useful, noninvasive tool in the diagnosis and management of eosinophilic asthma [2].And measurement of $\mathrm{nNO}$ may provide a similarly useful tool in the management of upper airway inflammation although measurement procedures have yet to be fully standardised and it currently remains largely a research tool [2] The influence factors of FeNO and nNO levels in AR patients were studied by domestic and foreign scholars, which were used in adults [3-5] and children [6-8], but the findings were not consistent. And in this area, domestic researches were relatively less. So we decided to measure the FeNO and $\mathrm{nNO}$ levels in healthy children and AR children, to analyze the influence factors of $\mathrm{FeNO}$ and $\mathrm{nNO}$ and to discuss the clinical significance of FeNO and nNO levels in diagnosing AR.

\section{Materials and Methods}

\section{General Materials}

Our data came from children who were treated in our department between January 2014 and September 2014. We did not select children who were $<5$ years of age, because they were too young to cooperate during the examination. Based on symptoms, physical examination, video laryngoscopy, skin prick testing and specific allergen screening (serum slgE) results, we collected 30 children with AR ( 24 boys and 6 girls). The AR children were 68-168 months of age. A questionnaire about clinical features of AR children (clinical symptoms, seasons, triggers, perceived allergy, parental history and individual medical history) had been completed by parents. Clinical symptoms of allergic rhinitis consisted of nasal obstruction, watery rhinorrhoea, itching, and sneezing. Each symptom score and sum of allergic symptoms score were measured using a visual analogue scale from none (score 0 ) to very severe (score 5).In the current study, the diagnostic criteria for AR met the 2008 updated version guide of the ARIA [9]. A mite allergen skin prick reagent kit (Recordation S.P.A., Italy) was used for skin prick testing ( $\geq++$ [positive]) and a special protein analyser test (Beckman Coulter, USA) was used for serum slgE ( $\geq 3$ [positive]). Fifteen healthy children (11 boys and 4 girls) were chosen during the same period. The healthy children were 62-163 months of age. They had no clinical symptoms of disease. The exclusion criteria included chronic lung disease, central hypoventilation syndrome, immune deficiency disease, diabetes, tuberculosis, asthma, systemic metabolic storage diseases, morbid obesity, a history of upper and/or lower airway surgery, and respiratory infection within 4 weeks. The study was approved by the Medical Ethics Committee. All of the children's guardians signed informed consent.

\section{Methods}

The nNO and FeNO levels were measured non-invasively using a NIOX MINO system (Aerocrine AB, Solna, Sweden). The ambient NO level was not limited. The NO level was expressed as parts per billion (ppb 1ppb=109).The FeNO and nNO levels were measured between 2:00 pm and 9:00 pm. The room temperature was maintained at 20-30 with a relative humidity of $20 \%-60 \%$. The environment was clean, and measurements were obtained away from windows, keeping dust and pollen outside, and avoiding volatile gases. Interruptions from mobile phones and other strong electromagnetic signals within $2 \mathrm{~m}$ were avoided. Subjects were instructed not to drink or eat during the 2 hours prior to NO testing, not to actively and passively smoke tobacco, and not to drink liquids containing caffeine. Subjects were also instructed not to eat foods which were rich in nitrogen, such as sausage, organ meats, lettuce and spinach. The children abstained from intensive physical activities on the day of the measurements.

Exhaled NO: Children were sitting and discharged residual lung gas, then exhaled gas to the instrument after a deep inspiration through the filter, using the on-line standardized single-breath 
technique at a constant mouth flow rate of $50 \mathrm{ml} / \mathrm{s}$ and holding the breath for 6 s. The instrument analysed the FeNO levels automatically.

Nasal NO: After resting for approximately $15 \mathrm{~min}$, children were seated with their mouths closed. An olive stuffy plug was placed on the right nostril, and children continued to breathe normally. The instrument continuously pumped nasal gas through the sampling tube at a flow rate of $2 \mathrm{ml} / \mathrm{s}$, which was held $2 \mathrm{~min}$. Then, the instrument analysed the nNO levels automatically.

\section{Statistics}

Statistical analysis was performed using SPSS software package (IBM SPSS statistics 20.0). Every observation index was assessed by a one-sample Kolmogorov-Smirnov test. Normally distributed data were expressed as the mean \pm Standard Deviation (SD). The median (25th and 75th percentiles) was used if data were not normally distributed. Comparisons between continuous variables were estimate with an independent samples test, Student's t-test or a Mann-Whitney test, depending on the distribution of the variables. Correlations of parametric and nonparametric variables were obtained using Pearson's regression analysis, Spearman's rank correlation tests, and multiple linear regression analysis. Categorical variables were compared with a Kruskal-Wallis test. A p value $<0.05$ was considered significant statistically.

\section{Results}

Comparing FeNO and nNO Levels between AR and Healthy Children

\begin{tabular}{|c|c|c|c|}
\hline Group & Number & FeNO (ppb) & nNO (ppb) \\
\hline AR & 30 & $46.0(31.0 ; 84.0)$ & $187.5(178.0 ; 201.0)$ \\
\hline Healthy children & 15 & $12.0(10.0 ; 16.0)$ & $52.0(22.0 ; 139.0)$ \\
\hline Z value & & -5.427 & -4.449 \\
\hline P value & & 0 & 0 \\
\hline
\end{tabular}

Table1: Comparing FeNO and nNO levels between AR and healthy children

The FeNO and nNO levels of children with AR and healthy children are reported in Table1, Figure1 and Figure2. Compared with healthy children, FeNO and nNO levels in children with AR were increased, and the difference was statistically significant. The two groups were matched for months of age $(Z=-1.831, P=0.067)$, gender $(\mathrm{Z}=0.140, \mathrm{P}=0.708)$, and body mass index $(\mathrm{Z}=-0.745, \mathrm{P}=0.456)$, in which the difference in the balancing test was not statistically significant.

Correlation Analysis of FeNO and nNO Levels' Influence Factors in AR Children

Correlation analysis showed that FeNO levels were positively correlated with months of age $(\mathrm{r}=0.796, \mathrm{P}=0.000)$, and height $(\mathrm{r}=0.828$, $\mathrm{P}=0.000$ ) (Figure 3 and Figure4). In AR children, FeNO levels were not significantly correlated with gender, Body Mass Index (BMI), each symptom (nasal obstruction, watery rhinorrhoea, itching and sneezing) score and sum of allergic symptoms score. The nNO levels were not significantly correlated with months of age, gender, height, weight, BMI, each symptom(nasal obstruction, watery rhinorrhea, itching and sneezing) score and sum of allergic symptoms score $(\mathrm{P}>0.05)$. Using multivariate linear regression analysis, FeNO levels were positively correlated with months of age ( partial regression coefficient $\mathrm{r}=0.677, \mathrm{t}=39.087, \mathrm{P}<0.01$ ), height (partial regression coefficient $\mathrm{r}=1.157, \mathrm{t}=41.456, \mathrm{P}<0.01$ ) and $\mathrm{nNO}$ levels (partial regression coefficient $\mathrm{r}=0.125, \mathrm{t}=22.203, \mathrm{P}<0.01)$. The regression equation: Y (FeNO levels, ppb) $=-198.966+1.157 \mathrm{X}$ (height, $\mathrm{cm}$ ) $+0.677 \mathrm{X}$ (months of age, $\mathrm{m}$ ) $+0.125 \mathrm{X}$ (nNO levels, ppb), $\mathrm{F}=5486.437$, $\mathrm{P}<0.01$.But multivariate linear regression analysis showed that $\mathrm{nNO}$ levels were not significantly correlated with months of age, gender, height, weight, BMI, each symptom (nasal obstruction, watery rhinorrhea, itching and sneezing)score and sum of allergic symptoms score.

\section{Discussion}

The role of NO in the airways is complex, possibly including antibacterial effects, pro-inflammatory effects, and regulation of blood flow and cilia beat frequency [2]. In the help of NOS, L-arginine which is used as a substrate produces NO. There are three types of NOS: Type I is named as nervous NOS (nNOS) or brain NOS (bNOS), which mainly distributes in the nerve and brain cells. Type II distributes in macrophages and cells of inflammatory reaction areas, which can be activated and becomes inducible NOS (iNOS). Type III is named as endothelial NOS (eNOS), which exists in the membrane of vascular endothelial cells. Type I and type III is also called constructive NOS (cNOS), which are continuously expressed but not impacted by the human body environment. The cNOS can produce a few NO. Endotoxin and pro- inflammatory cytokines can inducte iNOS to produce a large number of NO by nuclear factor-kappa B (NF-kB). And these NO are cytotoxic, which can aggravate the degree of inflammatory reaction. In healthy people, cNOS exists in the nasal mucosa, and iNOS is primarily expressed by sinus mucosa. FeNO is derived from the alveoli and bronchi. Far greater levels of nNO are produced in the upper respiratory tract than the lower respiratory tract. $\mathrm{nNO}$ is mainly derived from the sinuses, and to a lesser extent from the nasal mucosa [2]. Therefore, FeNO and nNO levels can objectively assess the severity of lower and upper respiratory tract inflammation. But in AR, many inflammatory cytokines are released, which result in over-expression of nasal mucosa's iNOS, then producing a large number of NO. About FeNO and nNO levels, their influence factors are complex and diverse. According to the findings of the current study, we discussed as follow 


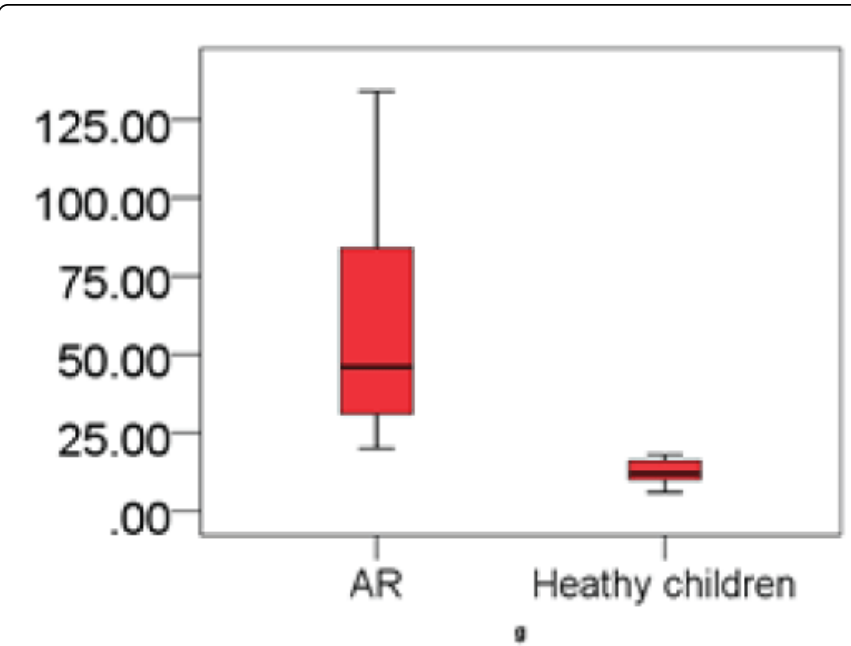

Figure 1: Comparision of FeNO levels in children with $\mathrm{AR}$ and Healthy children.

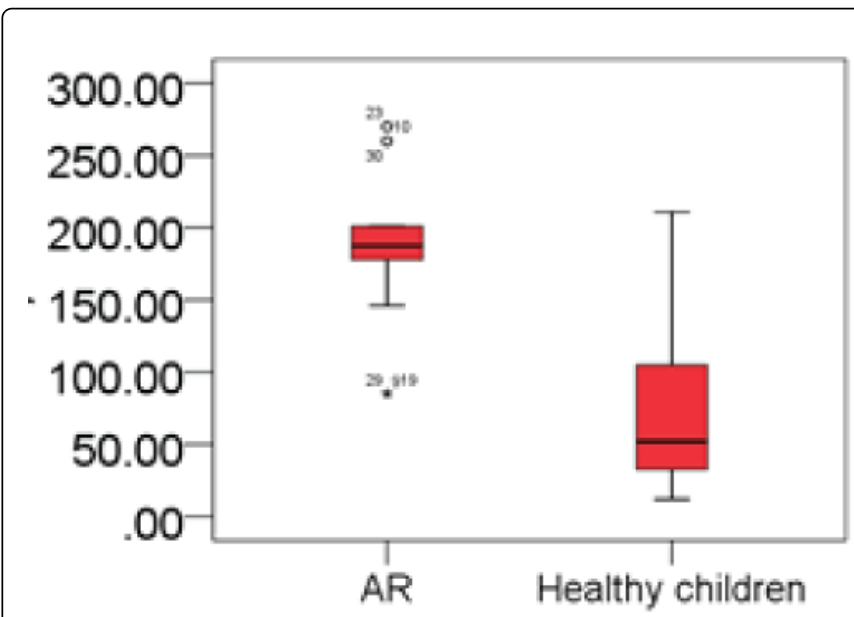

Figure 2: Comparision of nNO levels in children with $\mathrm{AR}$ and Healthy children.

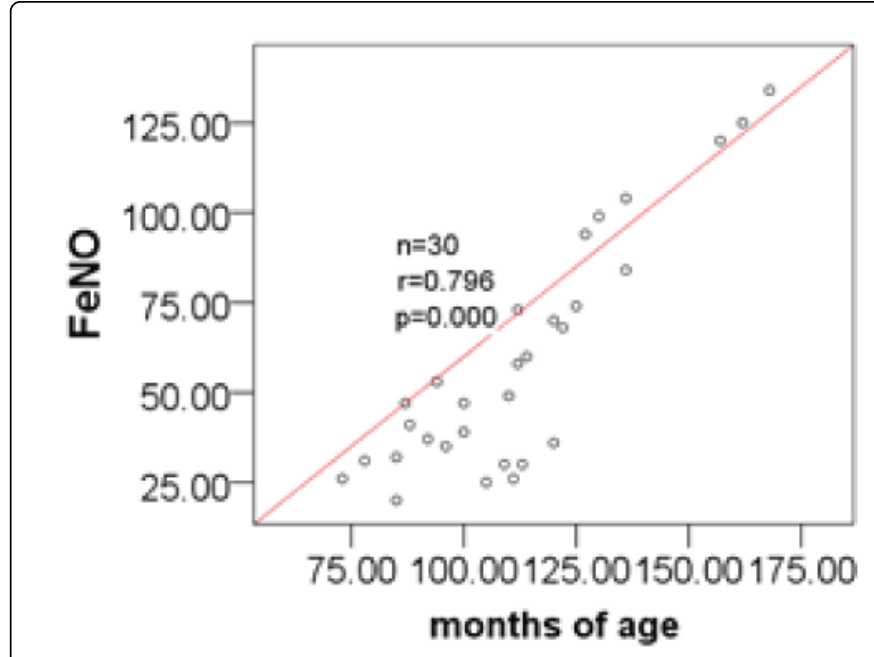

Figure 3: Correlation analysis between Feno levels and months of age in AR children.

We found that FeNO and nNO levels in AR children were was statistically significant higher than in healthy children, which was similar to Hung C's [6] and Baraldi's [7] studies. It showed that there was inflammation in AR patients' nasal and nNO levels could reflect the severity of upper inflammation. Our findings suggested that there were differently degrees inflammatory reaction in lower respiratory tract, but it did not reach the threshold of appearing clinical symptoms.

Lee et al [4] reported that there was significantly positive correlation between FeNO levels and watery rhinorrhea score, sneezing score, and sum of allergic symptoms score. Other symptoms scores were not significant correlation with FeNO levels. It was no significant correlation between nNO levels and any allergic symptoms scores. Lee's results [4] were similar to Baraldi's [7]. They thought the reasons needed to be further studied. Our data showed that FeNO, nNO levels were not significant correlation with each symptom (nasal obstruction, watery rhinorrhea, itching, and sneezing) score and sum of allergic symptoms score. Mechanism of the four symptoms (nasal obstruction, watery rhinorrhea, itching, and sneezing) are discussed as follow: they are nasal symptoms in early-onset phase of excitation stage in AR children. When allergen is interacted with mast cells or basophils off particle, inflammatory mediators (such as histamine, prostaglandins, leukotrienes, etc.) are relaeased. These mediators acted on sensory nerve endings, blood vessel walls and glands of nasal mucosa, and then nasal symptoms of early-onset phase were produced. Currently, further research is needed to know whether this pathogenesis is related with mechanism of FeNO and nNO. In addition, we need further studies to show whether the severity of allergic inflammation can be determined by these clinical symptoms. This is an aim that we explore the application of FeNO and nNO levels in AR. 
Page 4 of 4

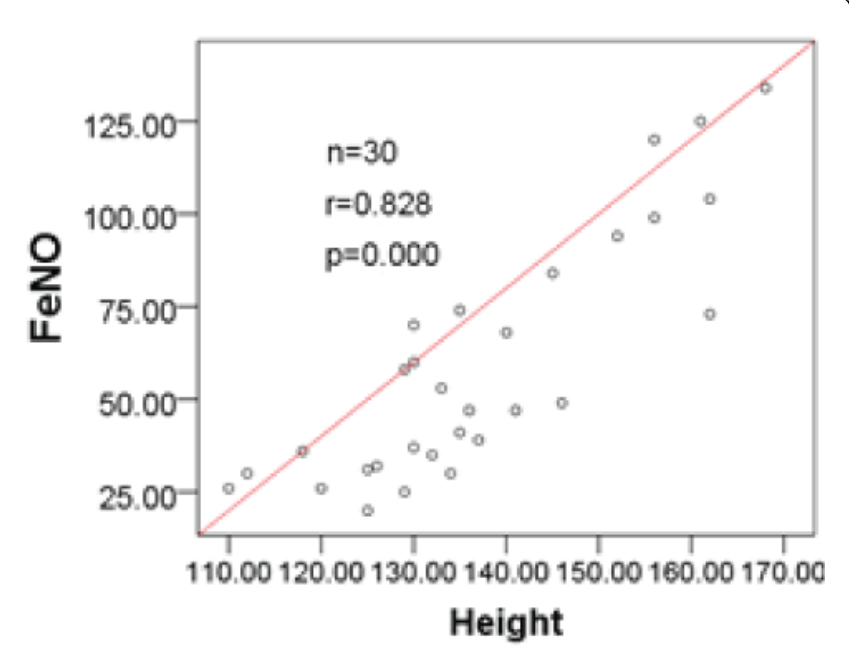

Figure 4: Correlation analysis between FeNo levels and height of age in AR children.

Williamson PA [5] reported FeNO levels were correlated with nNO levels in AR patients. Multivariate linear regression analysis showed that FeNO levels were correlated with years of age, gender, and the nNO levels after inhaling corticosteroids [5]. Our study showed that FeNO levels in AR children were positively correlated with months of age, height and nNO level. In AR children, the positive correlation between FeNO levels and nNO levels showed that AR was a risk factor for asthma. AR might be evolved into asthma, both of which diseases belonged to the concept of 'one airway one disease [9]. With age increasing, Children's height is gradually increasing, and then the total areas of respiratory tract mucosa are also increasing, especially in the lower respiratory tract [10]. Therefore, the epithelial cells of mucosa become more and more, while cytokine of the upper and lower airway inflammation are gradually increasing. Then iNOS of airway mucosa is significantly expressed, producing a large number of NO.Therefore, FeNO levels are positively related to months of age and height.

The shortcomings of this study: we didn't execute serum lgE testing and blood routine examination in every allergic rhinitis children, so we couldn't analyze the correlation between FeNO, nNO levels and eosinophils, allergen and total specific serum $\operatorname{lgE}$. It is difficult to thoroughly reveal clinical significance of $\mathrm{FeNO}$ and nNO levels in the diagnosis of AR.

\section{Conclusions}

Our data support that FeNO and nNO levels have a great value in diagnosing AR. And in AR without asthma, FeNO and nNO levels were simultaneous elevated.

\section{References}

1. Mahr TA, Malka J, Spahn JD (2013) Inflammoeometry in pediatric asthma: a review of fractional exhaled nitric oxide in clinical practice. Allergy Asthma Proc 34: 210-219.

2. Scadding G, Scadding G (2009) Update on the use of nitric oxide as a non-invasive measure of airways inflammation. Rhinology 47: 115-120.

3. Wilson AM, Dempsey OJ, Sims EJ, Lipworth BJ (2000) Subjective and objective markers of treatment response in patients with seasonal allergic rhinitis. Ann Allergy Asthma Immunol 85: 111-114.

4. Lee KJ, Cho SH, Lee S, K, HJ, et al. (2012) Nasal and exhaled nitric oxide in allergic rhinitis. J Clin Exp Otorhinolaryngol 5: 228-233.

5. Williamson PA, Vaidyanathan S, Clearie K, Stewart M, Lipworth BJ (2010) Relationship between fractional exhaled nitric oxide and nasal nitric oxide in airways disease. Ann Allergy Asthma Immunol 105: 162-167.

6. Hung CH, Hua YM, Hsu WT, Lai YS, Yang KD, et al. (2007) Montelukast decreased exhaled nitric oxide in children with perennial allergic rhinitis. J Pediatr Int 49: 322-327.

7. Baraldi E, Azzolin NM, Carra S, C Dario, L Marchesini, et al. (1988) Effect of topical steroids on nasal nitric oxide production in children with perennial allergic rhinitis: A pilot study. Respir Med 92: 558-561.

8. De Prins S, Marcucci F, Sensi L, Nelen V, Koppen G, et al. (2014) Exhaled nitric oxide and nasal tryptase are associated with wheeze, rhinitis and nasal allergy in primary school children. Biomarkers 19: 481-487.

9. Bousquet J, Khaltaev N, Cruz A, Fokkens WJ, Togias A, et al.(2008) Allergic rhinitis and its impact on asthma (ARIA) 2008 update (in collaboration with the World Health Organization,GA(2) LEN and AllerGen). Allergy 63: 8-160.

10. Olin AC, Rosengren A, Thelle DS, Torén K, Bake B, et al. (2006) Height, age, and Atopy are associated with fraction of exhaled nitric oxide in a large adult general population sample. Chest 130: 1319-1325. 